Alberta-Thy-26-94

\title{
The Affine symmetry of self-dual gravity
}

\author{
Viqar Husain* \\ Dept. of Mathematics and Statistics, University of Calgary, \\ Calgary, Alberta, Canada T2N 1N4.
}

\begin{abstract}
Self-dual gravity may be reformulated as the two dimensional principal chiral model with the group of area preserving diffeomorphisms as its gauge group. Using this formulation, it is shown that self-dual gravity contains an infinite dimensional hidden symmetry whose generators form the Affine (Kac-Moody) algebra associated with the Lie algebra of area preserving diffeomorphisms. This result provides an observable algebra and a solution generating technique for self-dual gravity.
\end{abstract}

PACS numbers: 02.20.Tw, 04.90.+e, 11.30.-j

*Address after September 15. '95: Center for Gravitational Physics and Geometry, The Pennsylvania State University, University Park, PA 16802-6300, USA. 


\section{INTRODUCTION}

Geometric field theories are of interest as models for quantum gravity. Topological field theories are examples of such theories that have a finite number of degrees of freedom. As such they are usually completely solvable quantum mechanically, and may be compared, in this respect, with the minisuperspace reductions of general relativity.

It is of more interest however to look for field theoretic models with an infinite number of degrees of freedom for modelling quantum gravity, since these are more likely to capture the main features of dynamical geometries. The types of models that have been studied include midisuperspace reductions of general relativity and self-dual gravity.

For the quantization of model theories, one approach involves finding a suitable Poisson algebra of observables at the classical level, which is to be represented as an operator algebra in the quantum theory. The problem of finding classical observables that form a nice algebra is not an easy task for a field theory, and most of the examples where this can be done are the integrable two dimensional theories, such as the KdV and Sine-Gordon equations, which are not geometric field theories in the sense required for quantum gravity.

In the cases where a suitable classical Poisson algebra can be found, quantization involves finding a representation of this algebra such that the observables are represented as Hermitian operators. This also may not be an easy task, especially if the Poisson algebra is infinite dimensional, which is expected to be the case for a field theory.

In this paper we study self-dual gravity as a possible model for quantum gravity. We will only consider in detail some classical aspects of the theory, and will outline an approach to quantization.

Self-dual gravity is defined by the statement that the Riemann curvature $R$ of a metric $g$ is proportional to its dual ${ }^{\star} R$ :

$$
{ }^{\star} R_{a b c d}:=\frac{1}{2} \epsilon_{a b}{ }^{e f} R_{e f c d}=\lambda R_{a b c d}
$$

where $\lambda$ is the proportionality factor and $\epsilon$ is the totally antisymmetric tensor density. $\lambda$ 
is determined by noting from the definition of duality that ${ }^{\star \star} R=\operatorname{det}\left(g^{a b}\right) R$. Therefore, taking the dual of ${ }^{\star} R=\lambda R$ gives

$$
{ }^{\star \star} R=\lambda^{\star} R=\lambda^{2} R \text {. }
$$

Hence $\lambda= \pm \sqrt{\operatorname{det}\left(g^{a b}\right)}$. This shows that self-dual gravity has non-flat solutions either for real metrics of signature $(--++)$ or $(++++)$, or for complex metrics.

Since the Riemann curvature may be split into its self-dual and anti-self-dual parts, imposing self-duality gives a reduction of general relativity with only one local degree of freedom. Self-duality implies the vanishing of the Ricci tensor via the cyclic Bianchi identity $R_{a[b c d]}=0:$

$$
R_{a c}=R_{a b c d} g^{b d}=\frac{1}{2 \lambda} \epsilon_{a b}^{e f} R_{e f c}^{b}=0 .
$$

This reduction therefore provides a non-trivial diffeomorphism invariant geometric field theory which may be studied classically and quantum mechanically.

An action principle, or equivalently a Hamiltonian formulation for self-dual gravity is not known, although the approach presented in this paper suggests a way of obtaining this.

Most work on this theory has been classical, and it is believed to be integrable. The main result that indicates integrability is Penrose's twistor construction of the general solution [1]. There are a number of other interesting results associated with this theory: There is a formulation using the Ashtekar Hamiltonian variables which give the self-duality equations in first order form as the evolution equations of triad of vector fields [2]; the latter form of the equations may be obtained from a $0+1$ reduction of the self-dual Yang-Mills equation [3]; the Plebanski equation [4], which is another formulation of the self-duality equation, may be derived from the two dimensional Wess-Zumino equation [5]; this latter work also identifies the infinite dimensional area preserving diffeomorphism algebra as a special symmetry associated with the theory.

Recently another formulation of self-dual gravity has been given by the author [6], which shows how this theory may be rewritten as the principal chiral model with the group of area 
preserving diffeomorphisms as its gauge group. Similar results have also been obtained by Ward [7]. This two dimensional formulation allows the use of a method [8] for obtaining an infinite number of conserved currents for self-dual gravity [9]. It thus provides another hint that the theory is integrable, since the principal chiral model with gauge group $S U(N)$ is known to be integrable [10]. It is likely that the two-dimensionsal techniques used to prove integrability will also be applicable to the infinite dimensional Lie algebra case relevant for self-dual gravity.

The chiral model formulation also provides an approach to obtaining a Hamiltonian formulation for self-dual gravity, since the Hamiltonian formulation of the chiral model for $S U(N)$ is known. However, again the generalization of this to the case of the infinite dimensional Lie algebra associated with self-dual gravity requires further study.

In this paper we will show that the conserved currents of self-dual gravity generate an infinite dimensional symmetry on the solution space of the theory, and that its generators form an Affine algebra associated with the Lie algebra of area preserving diffeomorphisms. This result identifies the observable algebra of self-dual gravity, since it is expected that symmetries on the solution space correspond to symmetries on the phase space generated via Poisson brackets.

( Infinite dimensional symmetry transformations on the solution space of two Killing field reduced general relativity were found by Geroch [11], and later shown to form an $\mathrm{SL}(2, \mathrm{R})$ Affine algebra [12]. Recently, the phase space realization of the solution space generators of the Geroch transformations have been found by the author [13].)

In the next Section we review the chiral model formulation of self-dual gravity. In Section III the symmetry transformations on the solution space are identified and their algebra is calculated. Section IV contains a summary and discussion of a possible approach to quantization. 


\section{SELF DUAL GRAVITY AS THE PRINCIPAL CHIRAL MODEL}

Self dual gravity may be reformulated as the chiral model in two dimensions [6]. This may be seen by starting with the Ashtekar-Jacobson-Smolin (AJS) form of the self-duality equations, which were derived from the Ashtekar Hamiltonian formulation of general relativity in Ref. [2]. The AJS equations are evolution equations for three divergence free vector fields $V_{i}^{a}(t, x, y, z)$ given on a three dimensional surface with a fixed volume element, which may be written as $d x \wedge d y \wedge d z$ in local coordinates. The indices $a, b \ldots$ are four dimensional, but in the following they will also be used on vectors that are tangent to three and two dimensional surfaces. The indices $i, j \ldots=1,2,3$ label the vector field. The AJS self-duality equations are

$$
\begin{gathered}
\partial_{a} V_{i}^{a}=0, \\
\frac{\partial V_{i}^{a}}{\partial t}=\frac{1}{2} \epsilon^{i j k}\left[V_{j}, V_{k}\right]^{a} .
\end{gathered}
$$

where $[$,$] is the Lie bracket, and the divergence in (2.1) is defined with respect to the fixed$ volume element on the three-surface. The self-dual metric is constructed from a solution of these equations using

$$
g^{a b}=(\operatorname{det} V)^{-1}\left[V_{i}^{a} V_{j}^{b} \delta^{i j}+V_{0}^{a} V_{0}^{b}\right]
$$

where we have defined $V_{0}^{a}=(\partial / \partial t)^{a}$. Eqn. (2.2) may be written in a covariant form as

$$
\left[V_{0}, V_{i}\right]^{a}=\epsilon^{i j k}\left[V_{j}, V_{k}\right]^{a}
$$

The principal chiral model is the two dimensional field theory whose Lagrangian is

$$
L=\frac{1}{2} \operatorname{Tr}\left(\partial_{\mu} g^{-1} \partial_{\nu} g\right) \eta^{\mu \nu}
$$

where $g(x, t)$ is a matrix group element and $\eta^{\mu \nu}(\mu, \nu=0,1=t, x)$ is a fixed flat background metric with signature $(++)$. The equations of motion are 


$$
\eta^{\mu \nu} \partial_{\mu}\left(g^{-1} \partial_{\nu} g\right)=0
$$

This equation may be written as two first order equations by using the Lie algebra valued 1-form $A_{\mu}:=g^{-1} \partial_{\mu} g:$

$$
\begin{gathered}
\eta^{\mu \nu} \partial_{\mu} A_{\nu}=0 \\
F_{\mu \nu}=\partial_{\mu} A_{\nu}-\partial_{\nu} A_{\mu}+\left[A_{\mu}, A_{\nu}\right]=0 .
\end{gathered}
$$

To see how Eqns. (2.4) may be rewritten as the chiral model equations (2.7 2.8) we first rewrite the former using the linear combinations

$$
\begin{array}{ll}
\mathcal{T}=V_{0}+i V_{1} & \mathcal{U}=V_{0}-i V_{1} \\
\mathcal{X}=V_{2}-i V_{3} & \mathcal{V}=V_{2}+i V_{3} .
\end{array}
$$

Then equations (2.4) become

$$
\begin{aligned}
& {[\mathcal{T}, \mathcal{X}]=[\mathcal{U}, \mathcal{V}]=0} \\
& {[\mathcal{T}, \mathcal{U}]+[\mathcal{X}, \mathcal{V}]=0 .}
\end{aligned}
$$

Using the coordinate freedom, we choose coordinates $t, x$ such that

$$
\mathcal{T}^{a}=\left(\frac{\partial}{\partial t}\right)^{a} \quad \mathcal{X}^{a}=\left(\frac{\partial}{\partial x}\right)^{a}
$$

with $\mathcal{U}$ and $\mathcal{V}$ arbitrary except that they satisfy the divergence free condition (2.1) with respect to the volume form $\omega=d x \wedge d y \wedge d z$ defined by the local coordinates. Since $\mathcal{T}^{a}$ and $\mathcal{X}^{a}$ commute, they are surface forming, and as we will see below, this surface will be the chiral model background.

The final step in obtaining the chiral model equations for self dual gravity is to make a specific choice for $\mathcal{U}$ and $\mathcal{V}$ without losing generality, such that these vector fields are divergence free (2.1), and lead to no reduction in the number of local degrees of freedom. A choice satisfying these conditions is 


$$
\begin{aligned}
& \mathcal{U}^{a}=\left(\frac{\partial}{\partial t}\right)^{a}+\alpha^{b a} \partial_{b} A_{0} \\
& \mathcal{V}^{a}=\left(\frac{\partial}{\partial x}\right)^{a}+\alpha^{b a} \partial_{b} A_{1}
\end{aligned}
$$

where

$$
\alpha^{a b}=\left(\frac{\partial}{\partial y}\right)^{[a} \otimes\left(\frac{\partial}{\partial z}\right)^{b]}=\left(\begin{array}{cc}
0 & 1 \\
-1 & 0
\end{array}\right)
$$

is the antisymmetric tensor that is the inverse of the two form $(d y \wedge d z)_{a b}$ in the $y-z$ surface, and $A_{0}(t, x ; y, z)$ and $A_{1}(t, x ; y, z)$ are two arbitrary functions. (A proof that there is no loss of generality in choosing $\mathcal{U}$ and $\mathcal{V}$ as in (2.13 2.14) is given in Ref. [9]. ) Substituting these into 2.102 .11 gives

$$
\begin{gathered}
\alpha^{a b} \partial_{b}\left[\partial_{0} A_{1}-\partial_{1} A_{0}+\left\{A_{0}, A_{1}\right\}\right]=0 \\
\alpha^{a b} \partial_{b}\left[\partial_{0} A_{0}+\partial_{1} A_{1}\right]=0 .
\end{gathered}
$$

where the bracket on the left hand side of equation (2.16) is the Poisson bracket with respect to $\alpha^{a b}$

$$
\left\{A_{0}, A_{1}\right\}:=\alpha^{a b} \partial_{a} A_{0} \partial_{b} A_{1}=\partial_{y} A_{0} \partial_{z} A_{1}-\partial_{z} A_{0} \partial_{y} A_{1}
$$

and $\partial_{0}, \partial_{1}$ denote partial derivatives with respect to $t, x$ etc. Equations (2.16-2.17) imply that the terms in their square brackets are equal to two arbitrary functions of $t$ and $x$, which we write as

$$
\begin{aligned}
\partial_{0} A_{1}-\partial_{1} A_{0}+\left\{A_{0}, A_{1}\right\} & =\partial_{0} F(t, x)+\partial_{1} G(t, x) \\
\partial_{0} A_{0}+\partial_{1} A_{1} & =\partial_{1} F(t, x)-\partial_{0} G(t, x),
\end{aligned}
$$

where $F, G$ are arbitrary functions of $t, x$. With the redefinitions

$$
a_{0}(t, x ; y, z):=A_{0}+G \quad a_{1}(t, x ; y, z):=A_{1}-F
$$

(2.192.20) become 


$$
\begin{array}{r}
f_{01}:=\partial_{0} a_{1}-\partial_{1} a_{0}+\left\{a_{0}, a_{1}\right\}=0, \\
\partial_{0} a_{0}+\partial_{1} a_{1}=0 .
\end{array}
$$

These are precisely the chiral model equations (2.7-2.8) on the $x, t$ 'spacetime', with $y, z$ treated as coordinates on an 'internal' space. The commutator in (2.8) has been replaced by the Poisson bracket (2.18). The gauge group is therefore the group of diffeomorphisms that preserve $\alpha^{a b}$ on the internal space, which is the group of area preserving diffeomorphisms. (We note that the redefinitions (2.21) do not alter the vector fields $\mathcal{U}$ and $\mathcal{V}$ in Eqns. (2.132.14) ).

\section{CONSERVED CURRENTS AND THE SYMMETRY ALGEBRA}

The chiral model for the $S U(N)$ groups, where the zero curvature equation (2.8) contains

a matrix commutator, is known to have an infinite dimensional hidden symmetry [8, 114]. Here we will show explicitly that when the matrix commutator is replaced by a Poisson bracket as in Eqn. (2.22), the hidden non-local symmetry transformations form an Affine algebra associated with the Lie algebra of area preserving diffeomorphisms.

We first define the covariant derivative acting on functions $\Lambda(t, x ; y, z)$ :

$$
D_{\mu} \Lambda:=\partial_{\mu} \Lambda+\left\{a_{\mu}, \Lambda\right\}
$$

where $\{$,$\} is as defined in (2.18) and \mu, \nu=0,1=t, x$ are the two dimensional 'spacetime' indices. Under the transformation

$$
\delta_{\Lambda} a_{\mu}:=D_{\mu} \Lambda
$$

the left hand sides of Eqns. (2.22 2.23$)$ change to

$$
\begin{gathered}
\delta_{\Lambda}\left(\partial_{\mu} a_{\mu}\right)=\partial_{\mu}\left(D_{\mu} \Lambda\right) \\
\delta_{\Lambda}\left(f_{01}\right)=\left\{f_{01}, \Lambda\right\} .
\end{gathered}
$$


Therefore, under the transformation (3.2), (2.22) is invariant because $f_{01}=0$, and (2.23) will be invariant only if $D_{\mu} \Lambda$ is a conserved current. We will now show following Refs. [8, 14], that a heirarchy of functions $\Lambda^{(n)}(t, x ; y, z)$ may indeed be constructed such that

$$
J_{\mu}^{(n)}:=D_{\mu} \Lambda^{(n)}
$$

are conserved currents.

We first note that for an arbitrary function $\Lambda^{(0)}(y, z)$, the current

$$
J_{\mu}^{(0)}:=D_{\mu} \Lambda^{(0)}=\left\{a_{\mu}, \Lambda^{(0)}\right\}
$$

is conserved by virtue of $(2.23)$. Therefore there exists a function $\Lambda^{(1)}(t, x ; y, z)$ such that

$$
J_{\mu}^{(0)}=\epsilon_{\mu \nu} \partial_{\nu} \Lambda^{(1)}
$$

where $\epsilon_{\mu \nu}$ is the antisymmetric tensor. We also have by Eqn. (2.23) that

$$
a_{\mu}=\epsilon_{\mu \nu} \partial_{\nu} \phi
$$

for some function $\phi(t, x ; y, z)$. Together these give

$$
\Lambda^{(1)}=\int_{-\infty}^{x} d x^{\prime} D_{0} \Lambda^{(0)}=\int_{-\infty}^{x} d x^{\prime}\left\{a_{0}, \Lambda^{(0)}\right\}=\left\{\phi, \Lambda^{(0)}\right\}
$$

We now define a second current by

$$
J_{\mu}^{(1)}:=D_{\mu} \Lambda^{(1)}
$$

It is straight forward to see that this is also conserved :

$$
\begin{aligned}
\partial_{\mu} J_{\mu}^{(1)} & =\partial_{\mu}\left(\left\{\partial_{\mu} \phi, \Lambda^{(0)}\right\}+\left\{a_{\mu},\left\{\phi, \Lambda^{(0)}\right\}\right\}\right) \\
& =\partial_{\mu}\left\{\epsilon_{\mu \nu} a_{\nu}, \Lambda^{(0)}\right\}+\left\{a_{\mu},\left\{\epsilon_{\mu \nu} a_{\nu}, \Lambda^{(0)}\right\}\right\} \\
& =\left\{f_{01}, \Lambda^{(0)}\right\}=0
\end{aligned}
$$

since $\partial_{\mu} \Lambda^{(0)}=0$ and $f_{01}=0$.

Assuming now that the $n$ th. current (3.5) is conserved implies that there is a function $\Lambda^{(n+1)}(t, x ; y, z)$ such that 


$$
J_{\mu}^{(n)}=\epsilon_{\mu \nu} \partial_{\nu} \Lambda^{(n+1)}
$$

Using this to show that $J_{\mu}^{(n+1)}:=D_{\mu} \Lambda^{(n+1)}$ is conserved will complete the induction:

$$
\begin{aligned}
\partial_{\mu} J_{\mu}^{(n+1)} & =D_{\mu} \partial_{\mu} \Lambda^{(n+1)}=D_{\mu} \epsilon^{\mu \nu} J_{\nu}^{(n)} \\
& =D_{\mu} \epsilon^{\mu \nu} D_{\nu} \Lambda^{(n)}=\left\{f_{01}, \Lambda^{(n)}\right\}=0
\end{aligned}
$$

Equations (3.5) and (3.12) give the relation between the successive $\Lambda^{(n)}$ :

$$
\Lambda^{(n+1)}(t, x)=\int_{-\infty}^{x} d x^{\prime} D_{0} \Lambda^{(n)}\left(t, x^{\prime}\right)
$$

The conserved currents generated by this procedure are non-trivial by construction. For if we are given a solution where the $a_{\mu}$ are not zero, then the method will give non-vanishing currents which are independent. This is because the $(n+1)$ th. current involves an extra integral which makes it more non-local than the $n$ th. one.

We would now like to see what the algebra of these currents is. The generators of the symmetry transformations $(3.2)$ are

$$
T_{\Lambda}^{(n)}:=\int d t d x\left(\delta_{\Lambda}^{(n)} a_{\mu}\right) \frac{\delta}{\delta a_{\mu}}=\int d^{2} x\left(D_{\mu} \Lambda^{(n)}\right) \frac{\delta}{\delta a_{\mu}},
$$

and they act on the space of solutions of (2.22 2.23) via

$$
\delta_{\Lambda}^{(n)} a_{\mu}=D_{\mu} \Lambda^{(n)}
$$

(Note that the generators $T_{\Lambda}^{(n)}$ defined here are functions of the 'internal' coordinates $(y, z)$. They could equally well be defined with additional integrals over $y$ and $z$, which would follow the standard textbook definitions of generators. All the results presented here go through with either definition because the $y, z$ internal space integrals are 'bystanders' in the calculations below.)

We recall that $a_{\mu}$ and $\Lambda^{(n)}$ are functions of all the coordinates $t, x, y, z$ for $n>0$. These functions may be expanded in a suitable orthonormal basis of functions $f_{\alpha}(y, z)$ on the 'internal' $y, z$ space as 


$$
\Lambda^{(n)}(t, x ; y, z)=\sum_{\alpha} f_{\alpha}(y, z) \Lambda_{\alpha}^{(n)}(t, x)
$$

where

$$
\Lambda_{\alpha}^{(n)}(t, x)=\int d y d z f_{\alpha}(y, z) \Lambda^{(n)}(t, x ; y, z)
$$

and $\alpha$ labels the basis. Such basis functions will satisfy a closed Poisson algebra

$$
\left\{f_{\alpha}, f_{\beta}\right\}=C_{\alpha \beta}^{\gamma} f_{\gamma}
$$

where $C_{\alpha \beta}^{\gamma}$ are the structure constants of the area preserving diffeomorphism group of the $y, z$ surface. For example, if the internal space is a torus, $y$ and $z$ are angles, and the natural basis are the functions $f_{m n}(y, z)=\exp [i(m y+n z)]$, with $\alpha \equiv(m, n)$. Their Poisson algebra is

$$
\left\{f_{m, n}, f_{p, q}\right\}=(p n-m q) f_{m+p, n+q}
$$

which identifies the structure constants. Natural bases for the area preserving diffeomorphisms for a number of surfaces have been studied in Ref. [15].

The generators (3.15) in the basis (3.18) may be written

$$
T_{\alpha}^{(n)}:=\int d^{2} x\left(\delta_{\alpha}^{(n)} a_{\mu}\right) \frac{\delta}{\delta a_{\mu}}=\int d^{2} x\left(D_{\mu} \Lambda_{\alpha}^{(n)}\right) \frac{\delta}{\delta a_{\mu}}
$$

and the task is to compute the commutators

$$
\begin{aligned}
{\left[T_{\alpha}^{(m)}, T_{\beta}^{(n)}\right]=} & \int d^{2} x \int d^{2} x^{\prime}\left[\delta_{\alpha}^{(m)} a_{\mu}(x) \frac{\delta}{\delta a_{\mu}(x)} D_{\nu} \Lambda_{\beta}^{(n)}\left(x^{\prime}\right) \frac{\delta}{\delta a_{\nu}\left(x^{\prime}\right)}\right. \\
& \left.-\delta_{\beta}^{(n)} a_{\nu}\left(x^{\prime}\right) \frac{\delta}{\delta a_{\nu}\left(x^{\prime}\right)} D_{\mu} \Lambda_{\alpha}^{(m)}(x) \frac{\delta}{\delta a_{\mu}(x)}\right] \\
= & \int d^{2} x D_{\mu}\left[\delta_{\alpha}^{(m)} \Lambda_{\beta}^{(n)}-\delta_{\beta}^{(n)} \Lambda_{\alpha}^{(m)}+\left\{\Lambda_{\alpha}^{(m)}, \Lambda_{\beta}^{(n)}\right\}\right] \frac{\delta}{\delta a_{\mu}}
\end{aligned}
$$

In the last equality we have assumed that the surface terms arising from integration by parts vanish. In the following we will show that the algebra of the $T_{\alpha}^{(n)}$ is the affine algebra $\left[T_{\alpha}^{(m)}, T_{\beta}^{(n)}\right]=C_{\alpha \beta}^{\gamma} T_{\gamma}^{(m+n)}$ for $m, n \geq 0$. 
We first note that $J_{\mu}^{(0)}:=\left\{a_{\mu}, \Lambda^{(0)}\right\}$ is a trivial current since its conservation is the equation of motion $\eta^{\mu \nu} \partial_{\mu} a_{\nu}=0$. Taking $\Lambda_{\alpha}^{(0)}(y, z):=f_{\alpha}(y, z)$, and using $(3.22)$ and $\delta_{\alpha}^{(n)} \Lambda_{\beta}^{(0)}=0$, (which follows because $\Lambda_{\beta}^{(0)}$ is independent of $a_{\mu}$ ), gives

$$
\left[T_{\alpha}^{(0)}, T_{\beta}^{(0)}\right]=C_{\alpha \beta}^{\gamma} T_{\gamma}^{(0)}
$$

Assuming now that

$$
\left[T_{\alpha}^{(0)}, T_{\beta}^{(n)}\right]=C_{\alpha \beta}^{\gamma} T_{\gamma}^{(n)}
$$

implies

$$
D_{\mu}\left(\delta_{\alpha}^{(0)} \Lambda_{\beta}^{(n)}\right)=C_{\alpha \beta}^{\gamma} D_{\mu} \Lambda_{\gamma}^{(n)}+\left\{D_{\mu} \Lambda_{\beta}^{(n)}, \Lambda_{\alpha}^{(0)}\right\}-\left\{\left\{a_{\mu}, \Lambda_{\alpha}^{(0)}\right\}, \Lambda_{\beta}^{(n)}\right\}
$$

Using the last equation and (3.14) then gives

$$
\left[T_{\alpha}^{(0)}, T_{\beta}^{(n+1)}\right]=C_{\alpha \beta}^{\gamma} T_{\gamma}^{(n+1)}
$$

The calculation of $\left[T_{\alpha}^{(1)}, T_{\beta}^{(1)}\right]$ requires $\delta_{\alpha}^{(1)} \Lambda_{\beta}^{(1)}$. In general for $n>0$ we have using (3.8), (3.9) and (3.14) that

$$
\delta_{\alpha}^{(n)} \Lambda_{\beta}^{(1)}=\left\{\delta_{\alpha}^{(n)} \phi, \Lambda_{\beta}^{(0)}\right\}=\left\{\int_{-\infty}^{x} d x^{\prime} D_{0} \Lambda_{\alpha}^{(n)}, \Lambda_{\beta}^{(0)}\right\}=\left\{\Lambda_{\alpha}^{(n+1)}, \Lambda_{\beta}^{(0)}\right\}
$$

Assuming that

$$
\left[T_{\alpha}^{(1)}, T_{\beta}^{(n)}\right]=C_{\alpha \beta}^{\gamma} T_{\gamma}^{(n+1)}
$$

implies

$$
\delta_{\alpha}^{(1)} \Lambda_{\beta}^{(n)}=C_{\alpha \beta}^{\gamma} \Lambda_{\gamma}^{(n+1)}+\left\{\Lambda_{\beta}^{(n+1)}, \Lambda_{\alpha}^{(0)}\right\}-\left\{\Lambda_{\alpha}^{(1)}, \Lambda_{\beta}^{(n)}\right\}
$$

Using this formula and (3.14) gives

$$
\begin{aligned}
\delta_{\alpha}^{(1)} \Lambda_{\beta}^{(n+1)} & =\int_{-\infty}^{x} d x\left[D_{0}\left(\delta_{\alpha}^{(1)} \Lambda_{\beta}^{(n)}\right)+\left\{D_{0} \Lambda_{\alpha}^{(1)}, \Lambda_{\beta}^{(n)}\right\}\right] \\
& =\int_{-\infty}^{x} d x\left[C_{\alpha \beta}^{\gamma} D_{0} \Lambda_{\gamma}^{(n+1)}+D_{0}\left\{\Lambda_{\beta}^{(n+1)}, \Lambda_{\alpha}^{(0)}\right\}-\left\{\Lambda_{\alpha}^{(1)}, D_{0} \Lambda_{\beta}^{(n)}\right\}\right] \\
& =C_{\alpha \beta}^{\gamma} \Lambda_{\gamma}^{(n+2)}+\left\{\Lambda_{\beta}^{(n+2)}, \Lambda_{\alpha}^{(0)}\right\}-\int_{-\infty}^{x} d x\left(\left\{\Lambda_{\alpha}^{(1)}, D_{0} \Lambda_{\beta}^{(n)}\right\}-\left\{\Lambda_{\beta}^{(n+1)},\left\{a_{0}, \Lambda_{\alpha}^{(0)}\right\}\right\}\right) \\
& =C_{\alpha \beta}^{\gamma} \Lambda_{\gamma}^{(n+2)}+\left\{\Lambda_{\beta}^{(n+2)}, \Lambda_{\alpha}^{(0)}\right\}-\left\{\Lambda_{\alpha}^{(1)}, \Lambda_{\beta}^{(n+1)}\right\} .
\end{aligned}
$$


It therefore follows from (3.22) that

$$
\left[T_{\alpha}^{(1)}, T_{\beta}^{(n+1)}\right]=C_{\alpha \beta}^{\gamma} T_{\gamma}^{(n+2)}
$$

which completes the induction. It remains to show that

$$
\left[T_{\alpha}^{(m)}, T_{\beta}^{(n)}\right]=C_{\alpha \beta}^{\gamma} T_{\gamma}^{(m+n)}
$$

for all $m, n$. This can again be proved by induction using (3.28) and the Jacobi identity. Assuming (3.32), we have

$$
\begin{aligned}
C_{\sigma \rho}^{\beta}\left[T_{\alpha}^{(n)}, T_{\beta}^{(m+1)}\right] & =\left[T_{\alpha}^{(n)},\left[T_{\sigma}^{(m)}, T_{\rho}^{(1)}\right]\right] \\
& =-\left[T_{\sigma}^{(m)},\left[T_{\rho}^{(1)}, T_{\alpha}^{(n)}\right]\right]-\left[T_{\rho}^{(1)},\left[T_{\alpha}^{(n)}, T_{\sigma}^{(m)}\right]\right] \\
& =-C_{\rho \alpha}^{\gamma}\left[T_{\sigma}^{(m)}, T_{\gamma}^{(n+1)}\right]+C_{\sigma \alpha}^{\gamma}\left[T_{\rho}^{(1)}, T_{\gamma}^{(m+n)}\right] \\
& =-C_{\rho \alpha}^{\gamma}\left[T_{\sigma}^{(m)}, T_{\gamma}^{(n+1)}\right]+C_{\sigma \alpha}^{\gamma} C_{\rho \gamma}{ }^{\beta} T_{\beta}^{(m+n+1)} .
\end{aligned}
$$

Therefore

$$
C_{\sigma \rho}^{\beta}\left[T_{\alpha}^{(n)}, T_{\beta}^{(m+1)}\right]+C_{\rho \alpha}^{\beta}\left[T_{\sigma}^{(m)}, T_{\beta}^{(n+1)}\right]=C_{\sigma \alpha}^{\beta} C_{\rho \beta}^{\gamma} T_{\gamma}^{(m+n+1)} .
$$

Expanding the second term on the r.h.s. gives

$$
\begin{aligned}
C_{\rho \alpha}^{\beta}\left[T_{\sigma}^{(m)}, T_{\beta}^{(n+1)}\right] & =-\left[T_{\rho}^{(n)},\left[T_{\alpha}^{(1)}, T_{\sigma}^{(m)}\right]\right]-\left[T_{\alpha}^{(1)},\left[T_{\sigma}^{(m)}, T_{\rho}^{(n)}\right]\right] \\
& =-C_{\alpha \sigma}^{\beta}\left[T_{\rho}^{(n)}, T_{\beta}^{(m+1)}\right]-C_{\sigma \rho}^{\beta} C_{\alpha \beta}^{\gamma} T_{\gamma}^{(m+n+1)} .
\end{aligned}
$$

Substituting this back into (3.34) gives

$$
C_{\sigma \rho}^{\beta}\left[T_{\alpha}^{(n)}, T_{\beta}^{(m+1)}\right]+C_{\sigma \alpha}^{\beta}\left[T_{\rho}^{(n)}, T_{\beta}^{(m+1)}\right]=\left(C_{\sigma \rho}^{\beta} C_{\alpha \beta}^{\gamma}+C_{\sigma \alpha}^{\beta} C_{\rho \beta}^{\gamma}\right) T_{\gamma}^{(m+n+1)}
$$

This completes the proof that the hidden symmetries of self-dual gravity form the Affine algebra associated with the Lie algebra of area preserving diffeomorphisms.

We note that for $m=n=0$, the algebra (3.32) is that of area preserving diffeomorphisms, or $w_{\infty}$, which is the special case identified previously [5]. We note also that there is an infinite dimensional commuting set of generators obtained by setting the Lie algebra indices 
in (3.32) to be equal: $\alpha=\beta$. This commuting set may provide the proof of integrability in the Liouville sense, if it can be shown that it also arises from two distinct Hamiltonian formulations in the manner standard for two dimensional integrable models [16].

The symmetry transformations that we have found also provide a solution generating technique, whereby given one solution, there is a method for obtaining a new solution. This is analogous to the procedure given by Geroch for two Killing field reductions of general relativity [1]].

We consider a one parameter set of solutions $a_{\mu}(s ; t, x ; y, z)$ such that $s=0$ is the given solution. Then a new solution, specified by the parameter value $s=1$, is obtained by integrating the equation

$$
\frac{d a_{\mu}}{d s}=F_{n}^{\alpha}(s) \delta_{\alpha}^{(n)} a_{\mu}=F_{n}^{\alpha}(s) D_{\mu} \Lambda_{\alpha}^{(n)}
$$

where $F_{n}^{\alpha}(s)$ are arbitrary functions which will characterize the new solution. (There is an implied sum over repeated indices).

This procedure for generating solutions is in fact general, and applies to any theory where hidden (non-gauge) symmetries exist on the solution space. If we consider the Hamiltonian formulation of the self-dual gravity chiral model, the phase space analog of (3.37) would involve writing the hidden symmetry generators as functionals of the phase space variables, and replacing $\delta_{\alpha}^{(n)} a_{\mu}$ in (3.37) by the Poisson bracket.

\section{DISCUSSION}

We have studied self-dual gravity in its formulation as the two dimensional principal chiral model, and obtained a result at the classical level which identifies the observable algebra for this theory.

There are two main questions that remain for the classical theory: (1) Can the Hamiltonian formulation of the $S U(N)$ chiral model be generalized to the infinite dimensional Lie algebra case relevant for self-dual gravity, and (2) can the phase space analogs of the 
Affine symmetry generators (3.21) be determined in the Hamiltonian formulation of this chiral model? Here the Affine algebra should arise as the Poisson algebra of its phase space generators.

For addressing the quantization problem, one is led to the problem of finding representations of the Affine algebra associated with the Lie algebra of area preserving diffeomorphisms. The only representations that have been studied are those for $S U(N)$ [17], so this appears to be an open problem.

In the canonical approach to the quantization of general relativity motivated by the Ashtekar variables [18, knot theory enters in the characterization of physical states [19]. This is due essentially to the imposition of the spatial diffeomorphism constraint on functions of loops. Because of this, any theory that contains a three dimensional spatial diffeomorphism constraint will have physical states labelled by knot classes. An example of such a theory is given in Ref. 20.

Since self-dual gravity is a diffeomorphism invariant theory, one can ask how the connection with knot theory arises for this case. In the chiral model formulation discussed here, we have made some coordinate choices and hence fixed some of the diffeomorphism freedom. Therefore the connection with knot theory cannot arise in the same way as in [19]. Nevertheless it seems possible that a connection occurs via a different route. In the standard approach to the quantum integrability of two dimensional models, the Yang-Baxter equation arises. This equation is connected with knot theory in that it contains the Reidmeister moves, and furthermore, it provided the first instances of quantum group structures in physical problems [21]. Since the $S U(N)$ chiral model is integrable classically and quantum mechanically, it is possible that the chiral model approach to self-dual gravity will provide connections to quantum groups and knot theory in a similar way. The main issue that needs to be addressed for this is to see how, if at all, the presence of an infinite dimensional Lie algebra affects the integrability procedures for two dimensional models.

This work was supported by the Natural Science and Engineering Research Council of 
Canada. 


\section{REFERENCES}

[1] R. Penrose, Gen. Rel. and Grav. 7, 31 (1976).

[2] A. Ashtekar, T. Jacobson, and L. Smolin, Comm. Math. Phys. 115, 631 (1988).

[3] L. Mason and E. T. Newman, Comm. Math. Phys. 121, 659 (1989).

[4] J. Plebanski, J. Math. Phys. 16, 2395 (1975).

[5] Q. H. Park, Phys. Lett. B236, 429 (1990); Phys. Lett. B238, 287 (1990).

[6] V. Husain, Phys. Rev. Lett. 72, 800 (1994).

[7] R. S. Ward, Class. Quantum Grav. 7, L217 (1990).

[8] E. Brezin, C. Itzykson, J. Zinn-Justin, and J. B. Zuber, Phys. Lett. B82, 442 (1979).

[9] V. Husain, Class. Quantum Grav. 11, 927 (1994).

[10] L. D. Faddeev and L. A. Takhtajan, Hamiltonian methods in the theory of solitons, Springer (Berlin 1987).

[11] R. Geroch, J. Math. Phys. 13, 394 (1971).

[12] Y. S. Wu and M. L. Ge, J. Math. Phys. 24, 1187 (1983).

[13] V. Husain, 'Einstein's equations and the chiral model' submitted to Phys. Rev. D (1995).

[14] L. Dolan, Phys. Rev. Lett. 47, 1371 (1981).

[15] C. N. Pope and L. J. Romans, Class. Quantum Grav. 7, 97 (1990).

[16] See for example, A. Das, Integrable models, World Scientific (Singapore1989).

[17] For a review see P. Goddard and D. Olive, Intern. Jour. Mod. Phys. A1, 303 (1986).

[18] A. Ashtekar, Phys. Rev. Lett. 57, 2244 (1986); Phys. Rev. D36, 1587 (1987); Nonperturbative canonical gravity, (World Scientific, Singapore, 1991). 
[19] C. Rovelli and L. Smolin, Phys. Rev. Lett. 61, 1155 (1988); Nucl. Phys. B331, 80 (1990).

[20] V. Husain and K. V. Kuchar, Phys. Rev. D 42, 4070 (1990).

[21] L. Kauffman, Knots and Physics 2nd. Ed., World Scientific (Singapore 1994). 